\title{
SOME BLACKWELL-TYPE RENEWAL THEOREMS FOR WEIGHTED RENEWAL FUNCTIONS
}

\author{
JIANXI LIN, ${ }^{*}$ Xiamen University
}

\begin{abstract}
In this paper, a new approach is proposed to investigate Blackwell-type renewal theorems for weighted renewal functions systematically according to which of the tails of weighted renewal constants or the underlying distribution is asymptotically heavier. Some classical results are improved considerably.

Keywords: Weighted renewal function; renewal theory; Blackwell's theorem; regular oscillation

2000 Mathematics Subject Classification: Primary 60K05
\end{abstract}

Secondary 60E05; 26A12

\section{Introduction}

Assume that $X$ is a nonnegative random variable with distribution function $F$ and finite mean $\mu$. Moreover, $\left\{X_{n}\right\}_{n \geq 1}$ is a sequence of independent and identically distributed (i.i.d.) random variables having the same distribution as $X$. Let

$$
S_{0}:=0, \quad S_{n}:=X_{n}+S_{n-1}, \quad n \geq 1 .
$$

Here $\left\{S_{n}\right\}_{n \geq 0}$ denotes the renewal epochs. The renewal function $U(x)$ counts the average number of renewals up to time $x$ :

$$
U(x):=\sum_{n=0}^{\infty} F^{n *}(x) .
$$

Results about the asymptotic behavior of $U(x)$ as $x \rightarrow \infty$ are usually called renewal theorems. In particular, the famous Blackwell renewal theorem states that if $F$ is nonarithmetic then, for every fixed $h>0$,

$$
U(x+h)-U(x) \rightarrow \frac{h}{\mu} \quad \text { as } x \rightarrow \infty .
$$

Information on the classical renewal theorems can be found in standard textbooks such as [8] and [12].

Furthermore, the following weighted renewal function has been considered:

$$
G(x):=\sum_{n=0}^{\infty} a_{n} F^{n *}(x),
$$

where $\left\{a_{n}\right\}$ is a sequence of nonnegative numbers, named weighted constants. Obviously, if $a_{n} \equiv 1$ then $G$ reduces to the ordinary renewal function $U$, while if $a_{n}=1 / n, n \geq 1$, then $G$ is called a harmonic renewal function.

Received 30 April 2008; revision received 29 October 2008.

* Postal address: Mathematics School, Xiamen University, Xiamen, Fujian, 361005, P. R. China.

Email address: linjx999@gmail.com 
In this paper we focus on the Blackwell-type theorems for $G(x)$. According to which of the tails of weighted renewal constants or the underlying distribution is asymptotically heavier, we consider the following three cases:

Case 1: $\left\{a_{n}\right\}$ plays a dominant role;

Case 2: the tail of $F$ plays a dominant role;

Case 3: $\left\{a_{n}\right\}$ and the tail of $F$ play roles of equal importance.

A unified method is proposed to treat the three cases above and it is different from the generating function approach of, for example, [6].

In the case of subordination, Stam [19] was the first to consider the relation between $\left\{a_{n}\right\}$, the tail of $F$, and the tail of $G$, and also considered the three cases in the present paper. However, for the Blackwell-type theorems on $G$, the three cases were usually considered separately.

In connection with case $1, a_{n}=n^{\alpha}(\alpha \in \mathbb{R})$ was first considered in [11] and then it was generalized by Embrechts et al. [6] to the case in which $a_{n}$ is regularly varying. More generally, the case in which $a_{n}$ is regularly oscillating (for its definition, see Section 2 ) has been considered in [17]. Our results for case 1 (see Theorem 3.1 and its corollaries, below) unify and generalize most of these corresponding results. In particular, the monotonicity condition on $\left\{a_{n}\right\}$, which was required in [17], is dropped. Moreover, we also establish some reverse result for Theorem 3.1.

It should be noted that in Theorem 3.1 and its corollaries we have assumed the regular oscillation of $\left\{a_{n}\right\}$. For related results which do not require such an assumption, we refer the reader to [10] and [13]. Moreover, we also assume that the random variable $X$ is nonnegative. The real random variable $X$ and regularly varying weights $\left\{a_{n}\right\}$ have been considered by Alsmeyer [1]. Alsmeyer obtained best possible results relating moments of $X$ to $G(x)$ and $G(x+h)-G(x)$.

In connection with case 2, Chover et al. [5] established the Blackwell-type renewal theorem under the assumptions that $F$ satisfies some subexponential condition while $a_{n}$ has a nice generating function. Related results are also established in [2]. Our result for case 2 (see Theorem 3.3, below) extends these results by making the conditions on $F$ stronger and the conditions on $\left\{a_{n}\right\}$ weaker. Case 3 is first considered in the present paper.

For an introduction to weighted renewal theory, we refer the reader to [15].

In the sequel we mainly state the results and method in the case that $F$ is an arithmetic distribution. Results for the nonarithmetic case are appended in Section 6. Finally, some remarks are included in Section 7.

\section{Notation}

For two functions or number sequences $f$ and $g$, we write

- $f \sim g$ if $\lim (f / g)=1$;

- $f=o(g)$ if $\lim (f / g)=0$;

- $f \lesssim g$ or $f=O(g)$ if $\lim \sup (f / g)<\infty$;

- $f \gtrsim g$ if $\lim \inf (f / g)>0$;

- $f \asymp g$ if $0<\liminf (f / g) \leq \lim \sup (f / g)<\infty$. 
For any sequences of numbers $\left\{g_{m}\right\}$ and $\{f(\varepsilon, m)\}$ with parameter $\varepsilon$, by the notation

$$
f(\varepsilon, m)=o\left(g_{m}\right), \quad m \rightarrow \infty, \varepsilon \rightarrow 0+,
$$

and

$$
f(\varepsilon, m) \sim g_{m}, \quad m \rightarrow \infty, \varepsilon \rightarrow 0+,
$$

we have

$$
\lim _{\varepsilon \rightarrow 0+} \limsup _{m \rightarrow \infty}\left|\frac{f(\varepsilon, m)}{g_{m}}\right|=0
$$

and

$$
\lim _{\varepsilon \rightarrow 0+} \limsup _{m \rightarrow \infty}\left|\frac{f(\varepsilon, m)}{g_{m}}-1\right|=0,
$$

respectively.

With any sequence of numbers $\left\{g_{n}\right\}_{n \geq 0}$, we always associate a function $g$ in such a way that

$$
g(x):=g_{[x]},
$$

where, here and throughout the paper, $[x]$ denotes the integer part of $x$.

Definition 2.1. A sequence of nonnegative numbers $\left\{g_{n}\right\}$ is called regularly varying, denoted by $g \in R V(\alpha)$, where $\alpha \in \mathbb{R}$, if, for every fixed $y>0$,

$$
\frac{g(x y)}{g(x)} \rightarrow y^{\alpha} \quad \text { as } x \rightarrow \infty .
$$

Definition 2.2. A sequence of nonnegative numbers $\left\{g_{n}\right\}$ is called regularly oscillating, denoted by $g \in \mathfrak{C}$, if

$$
\lim _{\substack{x \rightarrow y \rightarrow \infty \\ x / y \rightarrow 1}} \frac{g(x)}{g(y)}=1 .
$$

Definition 2.3. A sequence of nonnegative numbers $\left\{g_{n}\right\}$ is called dominatedly varying, denoted by $g \in \mathfrak{D}$, if, for every fixed $y>0$,

$$
g(x y) \asymp g(x) \quad \text { as } x \rightarrow \infty .
$$

Definition 2.4. A sequence of nonnegative numbers $\left\{g_{n}\right\}$ is said to be long tailed, denoted by $g \in \mathfrak{L}$, if, for every fixed $y \in \mathbb{R}$,

$$
\lim _{x \rightarrow \infty} \frac{g(x+y)}{g(x)} \rightarrow 1 .
$$

Definition 2.5. A sequence of nonnegative numbers $\left\{g_{n}\right\}$ with $\sum_{n=0}^{\infty} g_{n}=1$ is said to be subexponential, denoted by $g \in S D$, if $g \in \mathfrak{L}$ and

$$
\frac{g_{n}^{2 *}}{g_{n}} \rightarrow 2 \text { as } n \rightarrow \infty,
$$

where $g^{2 *}$ denotes the two-fold convolution of $\left\{g_{n}\right\}$, that is

$$
g_{n}^{2 *}=\sum_{k=0}^{n} g_{n-k} g_{k} .
$$


For more properties of the classes $R V$ and $\mathfrak{D}$, we refer the reader to [4]. The class $\mathfrak{C}$ was introduced in [3] and the class $S D$ was introduced in [5].

It is well known that the following relation holds:

$$
R V \subset \mathfrak{C} \subset \mathfrak{D} \cap \mathfrak{L} .
$$

Furthermore, if $\left\{g_{n}\right\}$ satisfies $\sum_{n=0}^{\infty} g_{n}=1$ then

$$
g \in \mathfrak{D} \cap \mathfrak{L} \quad \Longrightarrow \quad g \in S D .
$$

Definition 2.6. A sequence of nonnegative numbers $\left\{g_{n}\right\}$ is said to be almost decreasing if

$$
\sup _{y \geq x} g(y) \asymp g(x) \quad \text { as } x \rightarrow \infty \text {. }
$$

For any function or sequence $f \in \mathfrak{D}$, its lower Matuszewska index is defined as

$$
\beta(f):=\frac{\sup _{\lambda>1}\left(\log f_{*}(\lambda)\right)}{\log \lambda}, \quad \text { where } \quad f_{*}(\lambda):=\liminf _{x \rightarrow \infty} \frac{f(\lambda x)}{f(x)} .
$$

By Corollary 2.1.6 of [4] we have

$$
\beta(f)=\frac{\lim _{\lambda \rightarrow \infty}\left(\log f_{*}(\lambda)\right)}{\log \lambda} .
$$

For more properties of almost monotonicity or the Matuszewska index, we refer the reader to [4, pp. 68-74].

Similar to that of distribution functions, we define the hazard rate of a sequence $\left\{g_{n}\right\}$ as

$$
q_{g}(n):=\frac{g_{n+1}-g_{n}}{g_{n}} \quad \text { if } g_{n}>0 .
$$

For a distribution $F$, we define

$$
\rho_{F}:=-\limsup _{x \rightarrow \infty} \frac{\log \bar{F}(x)}{\log x},
$$

where, here and throughout the paper, $\bar{F}=1-F$ denotes the tail of $F$.

\section{Main results}

In the following discussion we always assume that the distribution $F$ is arithmetic and, without loss of generality, its span is assumed to be 1; similar results can be obtained for the case in which the span of $F$ is not equal to 1 . Moreover, we always assume that the distribution $F$ has a finite mean $\mu$. Let

$$
p_{m}=\mathrm{P}(X=m), \quad m=0,1,2, \ldots,
$$

and, without loss of generality, we always assume that $p_{0}<1$.

Next, we will state the main results for the three cases separately. 


\subsection{The case in which $\left\{a_{n}\right\}$ plays a dominant role}

Theorem 3.1. Suppose that $a \in \mathfrak{C}$ and that

$$
\bar{F}(m)=o\left(\frac{a_{m}}{\sum_{n=1}^{m} a_{n}}\right) \text { as } m \rightarrow \infty .
$$

Then

$$
G(m)-G(m-1) \sim \frac{a(m / \mu)}{\mu} \text { as } m \rightarrow \infty .
$$

Corollary 3.1. Suppose that $a \in \mathfrak{C}$ and that $\beta(a)>-1$. Then relation (3.2) holds.

Corollary 3.2. Suppose that $a \in \mathfrak{C}, \beta(a) \leq-1$, and that there exists a constant $\beta<\beta(a)$ such that

$$
\bar{F}(m)=o\left(m^{\beta}\right) \quad \text { as } m \rightarrow \infty .
$$

Then relation (3.2) holds.

If $\sum_{n=1}^{m} a_{n}<\infty$ then condition (3.1) reduces to $\bar{F}(m)=o\left(a_{m}\right)$. However, by the proof of Theorem 3.1, such a condition can be further weakened. We reformulate such a result as follows.

Corollary 3.3. Suppose that $a \in \mathfrak{C}, \sum_{n=1}^{\infty} a_{n}<\infty, p_{m}=o\left(a_{m}\right)$, and that

$$
\bar{F}(m)=O\left(a_{m}\right) \text { as } m \rightarrow \infty .
$$

Then relation (3.2) holds.

It is easy to see that Theorem 3.1 implies that $G(m)-G(m-1) \in \mathfrak{C}$. Then the question that arises is: under what conditions does $G(m)-G(m-1) \in \mathfrak{C}$ imply that $a \in \mathfrak{C}$ ? We give an answer to this problem as follows.

Theorem 3.2. Suppose that $\{G(m)-G(m-1)\} \in \mathfrak{C},\left\{a_{n}\right\}$ is ultimately monotone, and that

$$
\bar{F}(m)=o\left(\frac{G(m)-G(m-1)}{G(m)}\right) \text { as } m \rightarrow \infty .
$$

Then $a \in \mathfrak{C}$ and relation (3.2) holds.

Remark 3.1. Similar to Theorem 3.1, some corollaries can be derived from Theorem 3.2. Moreover, a similar reverse result for Corollary 3.3 can be formulated easily.

\subsection{The case in which the tail of $F$ plays a dominant role}

Theorem 3.3. Suppose that $p \in \mathfrak{D} \cap \mathfrak{L},\left\{p_{m}\right\}$ is almost decreasing, $\rho_{F}>1$, and that

$$
a_{m}=o\left(p_{m}\right) \text { as } m \rightarrow \infty .
$$

Then $\sum_{n=1}^{\infty} n a_{n}<\infty$ and

$$
G(m)-G(m-1) \sim\left(\sum_{n=1}^{\infty} n a_{n}\right) p_{m} \quad \text { as } m \rightarrow \infty .
$$




\subsection{The case in which $\left\{a_{n}\right\}$ and the tail of $F$ play roles of equal importance}

Theorem 3.4. Suppose that $p \in \mathfrak{C},\left\{p_{m}\right\}$ is almost decreasing, $\rho_{F}>1$, and that there exists a constant $K \geq 0$ such that

$$
a_{m} \sim K p_{m} \quad \text { as } m \rightarrow \infty
$$

Then $\sum_{n=1}^{\infty} n a_{n}<\infty$ and

$$
G(m)-G(m-1) \sim\left(\sum_{n=1}^{\infty} n a_{n}\right) p_{m}+\frac{a(m / \mu)}{\mu} \text { as } m \rightarrow \infty .
$$

Remark 3.2. When $K=0$, the above result is a special case of Theorem 3.3.

By Theorem 3.4, it is easy to obtain the following corollary.

Corollary 3.4. Suppose that $p \in R V(\alpha), \alpha<-2$, and that there exists a constant $K \geq 0$ such that relation (3.7) holds. Then $\sum_{n=1}^{\infty} n a_{n}<\infty$ and

$$
G(m)-G(m-1) \sim\left(\sum_{n=1}^{\infty} n a_{n}+\frac{K}{\mu^{\alpha+1}}\right) p_{m} \quad \text { as } m \rightarrow \infty .
$$

\section{Preliminary lemmas}

The following lemmas are needed in our proofs.

Lemma 4.1. Assume that $g \in \mathfrak{D}$ and that $\beta(g)>-1$. Then

$$
\sum_{n \leq \varepsilon m} g_{n}=o\left(m g_{m}\right), \quad m \rightarrow \infty, \varepsilon \rightarrow 0+.
$$

Proof. The result can be easily proved by using Corollary 2.6.2 and Proposition 2.2.1 of [4].

By Theorem 2.1.7(ii) and Proposition 2.2.1 of [4], it is easy to obtain the following lemma.

Lemma 4.2. Suppose that $g \in \mathfrak{D}$. Then there exist constants $C>0$ and $v>-\beta(g)$ such that, for sufficiently large $x$,

$$
g(x) \geq C x^{-v} .
$$

Lemma 4.3. For every positive integer $k$, we have

$$
\sum_{n=0}^{k} \mathrm{P}\left(S_{n}=m\right) \leq \frac{1}{1-p_{0}} \mathrm{P}\left(S_{k} \geq m\right)
$$

and

$$
\sum_{n=k}^{\infty} \mathrm{P}\left(S_{n}=m\right) \leq \frac{1}{1-p_{0}} \mathrm{P}\left(S_{k} \leq m\right)
$$

Proof. In view of

$$
\left\{S_{n}=m\right\}=\left\{S_{n}=m, X_{n+1} \neq 0\right\} \cup\left\{S_{n}=m, X_{n+1}=0\right\},
$$


we have

$$
\begin{aligned}
\sum_{n=0}^{k} \mathrm{P}\left(S_{n}=m\right) & =\sum_{n=0}^{k} \mathrm{P}\left(S_{n}=m, X_{n+1} \neq 0\right)+\sum_{n=0}^{k} \mathrm{P}\left(S_{n}=m, X_{n+1}=0\right) \\
& :=I+I I .
\end{aligned}
$$

Since $\left\{S_{n}=m, X_{n+1} \neq 0\right\}$ for $n=0,1, \ldots, k$ is a collection of disjoint events, we have

$$
I=\mathrm{P}\left(\bigcup_{n=0}^{k}\left\{S_{n}=m, X_{n+1} \neq 0\right\}\right) \leq \mathrm{P}\left(\bigcup_{n=0}^{k}\left\{S_{n}=m\right\}\right) \leq \mathrm{P}\left(S_{k} \geq m\right),
$$

where the last step holds since $X$ is nonnegative. Since $\left\{S_{n}=m\right\}$ and $\left\{X_{n+1}=0\right\}$ are independent, we obtain

$$
I I=\sum_{n=0}^{k} \mathrm{P}\left(S_{n}=m\right) \mathrm{P}\left(X_{n+1}=0\right)=p_{0} \sum_{n=0}^{k} \mathrm{P}\left(S_{n}=m\right) .
$$

Combining (4.3), (4.4), and (4.5) gives

$$
\sum_{n=0}^{k} \mathrm{P}\left(S_{n}=m\right) \leq \mathrm{P}\left(S_{k} \geq m\right)+p_{0} \sum_{n=0}^{k} \mathrm{P}\left(S_{n}=m\right),
$$

which is equivalent to (4.1). Equation (4.2) can be proved similarly.

Lemma 4.4. For every fixed $\varepsilon>0$, the following relation holds:

$$
\sum_{m(1-\varepsilon) / \mu<n<m(1+\varepsilon) / \mu} \mathrm{P}\left(S_{n}=m\right) \rightarrow \frac{1}{\mu} \text { as } m \rightarrow \infty .
$$

Proof. By Blackwell's renewal theorem for $U$ we have

$$
U(m)-U(m-1) \rightarrow \frac{1}{\mu} \quad \text { as } m \rightarrow \infty .
$$

Note that

$$
\begin{aligned}
U(m)-U(m-1)= & \sum_{n=0}^{\infty} \mathrm{P}\left(S_{n}=m\right) \\
= & \sum_{n \leq m(1-\varepsilon) / \mu} \mathrm{P}\left(S_{n}=m\right)+\sum_{m(1-\varepsilon) / \mu<n<m(1+\varepsilon) / \mu} \mathrm{P}\left(S_{n}=m\right) \\
& +\sum_{n \geq m(1+\varepsilon) / \mu} \mathrm{P}\left(S_{n}=m\right) \\
:= & I_{u}+I I_{u}+I I I_{u} .
\end{aligned}
$$

By Lemma 4.3 we have

$$
I_{u} \leq \frac{1}{1-p_{0}} \mathrm{P}\left(S_{[m(1-\varepsilon) / \mu]} \geq m\right) \quad \text { and } \quad I I I_{u} \leq \frac{1}{1-p_{0}} \mathrm{P}\left(S_{[m(1+\varepsilon) / \mu]} \leq m\right) .
$$


Hence, by the law of large numbers we have, for every fixed $\varepsilon>0$,

$$
I_{u} \rightarrow 0 \quad \text { and } \quad I I I_{u} \rightarrow 0 \quad \text { as } m \rightarrow \infty .
$$

From (4.6), (4.7), and (4.8), the desired result follows.

Lemma 4.5. Suppose that $a \in \mathfrak{C}$.

(i) If

$$
\sum_{n \leq \varepsilon m} a_{n} \mathrm{P}\left(S_{n}=m\right)=o\left(a_{m}\right), \quad m \rightarrow \infty, \varepsilon \rightarrow 0+,
$$

then relation (3.2) holds.

(ii) If there exists a sequence of numbers $\left\{d_{m}\right\}$ such that

$$
\sum_{n \leq \varepsilon m} a_{n} \mathrm{P}\left(S_{n}=m\right) \sim d_{m}, \quad m \rightarrow \infty, \varepsilon \rightarrow 0+,
$$

then

$$
G(m)-G(m-1) \sim \frac{a(m / \mu)}{\mu}+d_{m} \quad \text { as } m \rightarrow \infty .
$$

Proof. Let $\varepsilon>0$. We have

$$
\begin{aligned}
G(m)-G(m-1)= & \sum_{n=0}^{\infty} a_{n} \mathrm{P}\left(S_{n}=m\right) \\
= & \sum_{n \leq \varepsilon m} a_{n} \mathrm{P}\left(S_{n}=m\right)+\sum_{\varepsilon m<n \leq m(1-\varepsilon) / \mu} a_{n} \mathrm{P}\left(S_{n}=m\right) \\
& +\sum_{m(1-\varepsilon) / \mu<n<m(1+\varepsilon) / \mu} a_{n} \mathrm{P}\left(S_{n}=m\right)+\sum_{n \geq m(1+\varepsilon) / \mu} a_{n} \mathrm{P}\left(S_{n}=m\right) \\
:= & I_{a}+I I_{a}+I I I_{a}+I V_{a} .
\end{aligned}
$$

Note that

$$
I V_{a}=\sum_{n \geq m(1+\varepsilon) / \mu} a_{n} \mathrm{P}\left(S_{n}=m\right) \leq \sum_{n \geq m(1+\varepsilon) / \mu} a_{n} \mathrm{P}\left(S_{n} \leq \frac{n \mu}{1+\varepsilon}\right) .
$$

By the Cramér-Chernoff theorem we know that, as $n \rightarrow \infty, \mathrm{P}\left(S_{n} \leq n \mu /(1+\varepsilon)\right)$ tends to 0 at an exponential rate and, thus, by Proposition 2.2.1 of [4], $a_{n} \mathrm{P}\left(S_{n} \leq n \mu /(1+\varepsilon)\right)$ also tends to 0 at an exponential rate, while, by Lemma 4.2 , there exist positive constants $C>0$ and $v_{0}>\max \{-\beta(a), 0\}$ such that, for sufficiently large $m$,

$$
a_{m} \geq \mathrm{Cm}^{-v_{0}}
$$

hence, for every fixed $\varepsilon>0$,

$$
I V_{a}=o\left(a_{m}\right) \quad \text { as } m \rightarrow \infty .
$$

Note that $a \in \mathfrak{C}$; so by Lemma 4.4 we have

$$
I I I_{a} \sim a\left(\frac{m}{\mu}\right) \sum_{m(1-\varepsilon) / \mu<n<m(1+\varepsilon) / \mu} \mathrm{P}\left(S_{n}=m\right) \sim \frac{a(m / \mu)}{\mu}, \quad m \rightarrow \infty, \varepsilon \rightarrow 0+.
$$


By Theorem 2.0.8 of [4] and Lemma 4.3, we have, for every fixed $\varepsilon>0$,

$$
\begin{aligned}
I I_{a} & \asymp a_{m} \sum_{\varepsilon m<n \leq m(1-\varepsilon) / \mu} \mathrm{P}\left(S_{n}=m\right) \\
& \leq \frac{1}{1-p_{0}} a_{m} \mathrm{P}\left(S_{[m(1-\varepsilon) / \mu]} \geq m\right) \\
& =o\left(a_{m}\right) \quad \text { as } m \rightarrow \infty,
\end{aligned}
$$

where in the last step we have used the law of large numbers. Hence, from (4.10), (4.12), (4.13), and (4.14), the desired result follows.

Lemma 4.6. Assume that $a \in \mathfrak{D}$. Let $k_{m}$ be a nonnegative integer-valued and nondecreasing function of $m$. If

$$
\sum_{k_{m} \leq n \leq \varepsilon m}\left|a_{n}-a_{n+1}\right| \mathrm{P}\left(S_{n}>m\right)=o\left(a_{m}\right), \quad m \rightarrow \infty, \varepsilon \rightarrow 0+,
$$

then

$$
\sum_{k_{m} \leq n \leq \varepsilon m} a_{n} \mathrm{P}\left(S_{n}=m\right)=o\left(a_{m}\right), \quad m \rightarrow \infty, \varepsilon \rightarrow 0+.
$$

Proof. Without loss of generality, we assume that $\varepsilon \in(0,1)$. Let

$$
c_{n}:=\sum_{k=1}^{n} \mathrm{P}\left(S_{k}=m\right) .
$$

It is obvious that

$$
\begin{aligned}
\sum_{k_{m} \leq n \leq \varepsilon m} a_{n} \mathrm{P}\left(S_{n}=m\right) & =\sum_{k_{m} \leq n \leq \varepsilon m} a_{n}\left(c_{n}-c_{n-1}\right) \\
& \leq \sum_{k_{m} \leq n \leq \varepsilon m-1}\left(a_{n}-a_{n+1}\right) c_{n}+a(\varepsilon m) c(\varepsilon m) .
\end{aligned}
$$

By Lemma 4.3,

$$
c_{n} \leq \frac{1}{1-p_{0}} \mathrm{P}\left(S_{n} \geq m\right)
$$

hence, by condition (4.15) we know that

$$
\begin{aligned}
\left|\sum_{k_{m} \leq n \leq \varepsilon m-1}\left(a_{n}-a_{n+1}\right) c_{n}\right| & \leq \sum_{k_{m} \leq n \leq \varepsilon m-1}\left|a_{n}-a_{n+1}\right| c_{n} \\
& \leq \frac{1}{1-p_{0}} \sum_{k_{m} \leq n \leq \varepsilon m-1}\left|a_{n}-a_{n+1}\right| \mathrm{P}\left(S_{n} \geq m\right) \\
& \leq \frac{1}{1-p_{0}} \sum_{k_{m-1} \leq n \leq \varepsilon(m-1)}\left|a_{n}-a_{n+1}\right| \mathrm{P}\left(S_{n}>m-1\right) \\
& =o\left(a_{m-1}\right) \quad \\
& =o\left(a_{m}\right), \quad m \rightarrow \infty, \varepsilon \rightarrow 0+.
\end{aligned}
$$


Note that $a \in \mathfrak{D}$; hence, by (4.16),

$$
a(\varepsilon m) c(\varepsilon m)=o\left(a_{m}\right), \quad m \rightarrow \infty, \varepsilon \rightarrow 0+.
$$

Thus, the desired result is proved.

Lemma 4.7. If $a \in \mathfrak{D}$ then there exists a positive sequence $\left\{b_{n}\right\}$ with

$$
\limsup _{m \rightarrow \infty}\left|m q_{b}(m)\right|<\infty
$$

such that

$$
b_{m} \asymp a_{m} \quad \text { as } m \rightarrow \infty,
$$

and, thus,

$$
\beta(b)=\beta(a) .
$$

Proof. By the representation theorem for $\mathfrak{D}$ (see Theorem 2.2.7 of [4]), there exist two functions $\eta$ and $\xi$ which are bounded on some $[X, \infty)$, and measurable such that

$$
a(x)=\exp \left\{\eta(x)+\int_{1}^{x} \frac{\xi(t)}{t} \mathrm{~d} t\right\}, \quad x \geq 1 .
$$

Define a positive sequence as follows:

$$
b_{m}:=\exp \left\{\int_{1}^{m} \frac{\xi(t)}{t} \mathrm{~d} t\right\}, \quad m \geq 0 .
$$

Then, obviously, relation (4.18) holds and, thus, by (2.1), it is easy to obtain (4.19). Note that

$$
q_{b}(m)=\exp \left\{\int_{m}^{m+1} \frac{\xi(t)}{t} \mathrm{~d} t\right\}-1 .
$$

Then relation (4.17) follows easily from the boundedness of $\xi$.

Lemma 4.8. Assume that, for every fixed $n \geq 1$,

$$
\mathrm{P}\left(S_{n}=m\right)=o\left(a_{m}\right) \quad \text { as } m \rightarrow \infty .
$$

Let $\left\{b_{n}\right\}$ be a sequence satisfying (4.18). Then relation (4.9) holds if and only if relation (4.9) holds with the sequence $b$ in place of $a$.

Proof. Assume that relation (4.9) holds. By (4.18), there exist a positive integer $m_{0}$ and two constants $C_{1}, C_{2}>0$ such that, for all $m \geq m_{0}$,

$$
C_{1} a_{m} \leq b_{m} \leq C_{2} a_{m} .
$$

Therefore,

$$
\begin{aligned}
\sum_{m_{0} \leq n \leq \varepsilon m} b_{n} \mathrm{P}\left(S_{n}=m\right) & \leq C_{2} \sum_{m_{0} \leq n \leq \varepsilon m} a_{n} \mathrm{P}\left(S_{n}=m\right) \\
& \leq C_{2} \sum_{n \leq \varepsilon m} a_{n} \mathrm{P}\left(S_{n}=m\right) \\
& =o\left(a_{m}\right) \\
& =o\left(b_{m}\right), \quad m \rightarrow \infty, \varepsilon \rightarrow 0+.
\end{aligned}
$$


From condition (4.20), it follows that

$$
\sum_{n<m_{0}} b_{n} \mathrm{P}\left(S_{n}=m\right)=o\left(a_{m}\right)=o\left(b_{m}\right) \quad \text { as } m \rightarrow \infty .
$$

From (4.21) and (4.22), we know that relation (4.9) continues to hold with $b$ in place of $a$. The converse can be proved similarly.

Lemma 4.9. Suppose that $a \in \mathfrak{D}$. Then relation (4.20) holds for every fixed $n \geq 1$ if and only if

$$
p_{m}=o\left(a_{m}\right) \quad \text { as } m \rightarrow \infty .
$$

Proof. The proof is standard and follows as in, for example, [5].

Lemma 4.10, below, was implied by Theorem 1 of [9] and was formulated in [16].

Lemma 4.10. Let $\left\{X_{k}, k \geq 1\right\}$ be a sequence of i.i.d. nonnegative random variables with common distribution function $F$ and finite expectation $\mu$. Then, for all $v>0, x>0$, and $n \geq 1$,

$$
\mathrm{P}\left(S_{n}>x\right) \leq n \bar{F}\left(\frac{x}{v}\right)+\left(\frac{\mathrm{e} \mu n}{x}\right)^{v} .
$$

Lemma 4.11. Suppose that $p \in \mathfrak{D},\left\{p_{n}\right\}$ is almost decreasing, and that $\rho_{F}>1$. Then, for every fixed $\gamma>\mu$, there exist constants $C_{1}, C_{2}>0$ such that, when $n$ is sufficiently large, the relation

$$
C_{1} n p_{m} \leq \mathrm{P}\left(S_{n}=m\right) \leq C_{2} n p_{m}
$$

holds for all $m \geq \gamma n$.

Proof. Let $m^{\prime}=m-n \mu$. Then $m \geq \gamma n(\gamma>\mu)$ implies that

$$
\tau m \leq m^{\prime} \leq m
$$

where $\tau=1-\mu / \gamma>0$. Hence, the relation

$$
p\left(m^{\prime}\right) \asymp p_{m} \quad \text { as } m \rightarrow \infty
$$

holds uniformly for $m \geq \gamma n$. Let $\xi$ be the number of summands $X_{k}, k=1,2, \ldots, n$, in the sum $S_{n}$ such that $X_{k} \geq m^{\prime} / \alpha^{4}$, where $\alpha=-\log p\left(m^{\prime}\right)$. Then

$$
\begin{aligned}
\mathrm{P}\left(S_{n}=m\right)= & \mathrm{P}\left(S_{n}-n \mu=m^{\prime}, \xi=0\right)+\mathrm{P}\left(S_{n}-n \mu=m^{\prime}, \xi=1\right) \\
& +\mathrm{P}\left(S_{n}-n \mu=m^{\prime}, \xi \geq 2\right) \\
:= & I_{s}+I I_{s}+I I I_{s} .
\end{aligned}
$$

Note that, for every $\theta \in(0,1)$,

$$
I_{s} \leq \mathrm{P}\left(S_{n}-n \mu \geq \theta m^{\prime}, \max _{1 \leq k \leq n} X_{k}<\frac{m^{\prime}}{\alpha^{4}}\right):=I(\theta) .
$$

Then by using the same reasoning as in the proof of Theorem 3.1 of [14] we prove that, for every fixed $\gamma^{\prime}>0$, there exists a sequence $\theta:=\theta\left(m^{\prime}\right)$ with $\theta \rightarrow 0$ as $m^{\prime} \rightarrow \infty$ such that the relation

$$
I(\theta)=o\left(p\left(m^{\prime}\right)\right) \quad \text { as } n \rightarrow \infty,
$$


holds uniformly for $m^{\prime} \geq \gamma^{\prime} n$ and, thus, for every fixed $\gamma>\mu$,

$$
I_{s}=o\left(p_{m}\right) \quad \text { as } n \rightarrow \infty
$$

holds uniformly for $m \geq \gamma n$. Moreover, the proof of Theorem 3.1 of [14] also implies that both

$$
I I I_{s}=o\left(n p\left(m^{\prime}\right)\right)=o\left(n p_{m}\right) \quad \text { as } n \rightarrow \infty
$$

and

$$
I I_{s} \asymp n p\left(m^{\prime}\right) \asymp n p_{m} \quad \text { as } n \rightarrow \infty
$$

hold uniformly for $m \geq \gamma n$. From (4.30), (4.31), and (4.32), it follows that, for every fixed $\gamma>\mu$, the relation $\mathrm{P}\left(S_{n}=m\right) \asymp n p_{m}, n \rightarrow \infty$, holds uniformly for $m \geq \gamma n$, as desired.

Lemma 4.12. Assume that $p \in \mathfrak{D} \cap \mathfrak{L}$, that $\left\{p_{m}\right\}$ is almost decreasing, that $\rho_{F}>1$, and that $\sum_{n=1}^{\infty} n a_{n}<\infty$. Then

$$
\sum_{n \leq \varepsilon m} a_{n} \mathrm{P}\left(S_{n}=m\right) \sim\left(\sum_{n=1}^{\infty} n a_{n}\right) p_{m}, \quad m \rightarrow \infty, \varepsilon \rightarrow 0+.
$$

Proof. Without loss of generality, we assume that $\varepsilon<1 / \mu$. By Lemma 4.11, there exist a sufficiently large integer $n_{0}$ and a constant $C>0$ such that, when $n_{0}<n \leq \varepsilon m$,

$$
\mathrm{P}\left(S_{n}=m\right) \leq C n p_{m}
$$

Hence,

$$
\frac{\sum_{n_{0}<n \leq \varepsilon m} a_{n} \mathrm{P}\left(S_{n}=m\right)}{p_{m}} \leq C \sum_{n_{0}<n \leq \varepsilon m} n a_{n} \leq C \sum_{n_{0}<n} n a_{n} .
$$

Since $\sum_{n=1}^{\infty} n a_{n}<\infty$, we obtain

$$
\limsup _{n_{0} \rightarrow \infty} \limsup _{m \rightarrow \infty}\left\{\frac{\sum_{n_{0}<n \leq \varepsilon m} a_{n} \mathrm{P}\left(S_{n}=m\right)}{p_{m}}\right\}=0 .
$$

On the other hand, since $\left\{p_{m}\right\}$ is a subexponential sequence, then by Theorem 1 of [5] we have, for every fixed $n_{0}>0$,

$$
\lim _{m \rightarrow \infty}\left\{\frac{\sum_{n \leq n_{0}} a_{n} \mathrm{P}\left(S_{n}=m\right)}{p_{m}}\right\}=\sum_{n \leq n_{0}} n a_{n} .
$$

By (4.34) and (4.35), we prove (4.33).

\section{Proofs}

The proofs of the main results stated in Section 3 are presented in this section.

Proof of Theorem 3.1. By Lemma 4.5, it is sufficient to prove (4.9). Let $\left\{b_{n}\right\}$ be as in Lemma 4.7. By (4.18), there exist two constants $C_{1}$ and $C_{2}$ and a positive integer $n_{0}$ such that, for all $n \geq n_{0}$,

$$
C_{1} a_{n} \leq b_{n} \leq C_{2} a_{n},
$$


and, thus, for all $m \geq n_{0}$,

$$
C_{1} \sum_{n=n_{0}}^{m} a_{n} \leq \sum_{n=n_{0}}^{m} b_{n} \leq C_{2} \sum_{n=n_{0}}^{m} a_{n}
$$

Hence,

$$
\sum_{n=1}^{m} a_{n} \asymp \sum_{n=n_{0}}^{m} a_{n} \asymp \sum_{n=n_{0}}^{m} b_{n} \asymp \sum_{n=1}^{m} b_{n} \quad \text { as } m \rightarrow \infty .
$$

Therefore, by (3.1) we obtain

$$
\bar{F}(m)=o\left(\frac{b_{m}}{\sum_{n=1}^{m} b_{n}}\right) \quad \text { as } m \rightarrow \infty .
$$

By (4.17), there exists a constant $M \in(0, \infty)$ such that, for all $n \geq 0$,

$$
\left|n q_{b}(n)\right| \leq M
$$

Hence,

$$
\begin{aligned}
\sum_{n \leq \varepsilon m}\left|b_{n}-b_{n+1}\right| \mathrm{P}\left(S_{n}>m\right) & =\sum_{n \leq \varepsilon m} \frac{b_{n}\left|n q_{b}(n)\right| \mathrm{P}\left(S_{n}>m\right)}{n} \\
& \leq M \sum_{n \leq \varepsilon m} \frac{b_{n} \mathrm{P}\left(S_{n}>m\right)}{n}
\end{aligned}
$$

Let $v_{0}$ be as in (4.11). By Lemma 4.10, for all $m, n \geq 1$,

$$
\mathrm{P}\left(S_{n}>m\right) \leq n \bar{F}\left(\frac{m}{2 v_{0}}\right)+\left(\frac{\mathrm{e} \mu n}{m}\right)^{2 v_{0}} .
$$

Applying (5.3) to the right-hand side of (5.2), we obtain

$$
\begin{gathered}
\sum_{n \leq \varepsilon m}\left|b_{n}-b_{n+1}\right| \mathrm{P}\left(S_{n}>m\right) \leq M \bar{F}\left(\frac{m}{2 v_{0}}\right) \sum_{n \leq \varepsilon m} b_{n}+M(\mathrm{e} \mu)^{2 v_{0}} m^{-2 v_{0}} \sum_{n \leq \varepsilon m} n^{2 v_{0}-1} b_{n} \\
:=I_{b}+I I_{b} .
\end{gathered}
$$

From (5.1), it is easy to see that, for every fixed $\varepsilon \in\left(0,1 /\left(2 v_{0}\right)\right)$,

$$
I_{b} \leq M \bar{F}\left(\frac{m}{2 v_{0}}\right) \sum_{n \leq m /\left(2 v_{0}\right)} b_{n}=o\left(b_{\left[m / 2 v_{0}\right]}\right)=o\left(b_{m}\right) \quad \text { as } m \rightarrow \infty .
$$

Note that $v_{0}>\max \{-\beta(a), 0\}$; hence, by (2.1) and (4.19), it is easy to see that

$$
\beta\left(\left\{n^{2 v_{0}-1} b_{n}\right\}\right)=2 v_{0}-1+\beta(b)=2 v_{0}-1+\beta(a)>v_{0}-1>-1 .
$$

So by Lemma 4.1 we obtain

$$
\sum_{n \leq \varepsilon m} n^{2 v_{0}-1} b_{n}=o\left(m^{2 v_{0}} b_{m}\right), \quad m \rightarrow \infty, \varepsilon \rightarrow 0+.
$$


Hence,

$$
I I_{b}=o\left(b_{m}\right), \quad m \rightarrow \infty, \varepsilon \rightarrow 0+.
$$

From (5.4), (5.5), and (5.6), it follows that

$$
\sum_{n \leq \varepsilon m}\left|b_{n}-b_{n+1}\right| \mathrm{P}\left(S_{n}>m\right)=o\left(b_{m}\right), \quad m \rightarrow \infty, \varepsilon \rightarrow 0+.
$$

Hence, by Lemma 4.6 with $k_{m} \equiv 0$, we know that relation (4.9) holds with $b$ in place of $a$. From (3.1), it is easy to see that

$$
p_{m} \leq \bar{F}(m-1)=o\left(a_{m-1}\right)=o\left(a_{m}\right) \quad \text { as } m \rightarrow \infty .
$$

Hence, by Lemma 4.9 we know that condition (4.20) is satisfied. Therefore, by Lemma 4.8 we prove that relation (4.9) holds and, thus, by Lemma 4.5(i) we obtain the desired result.

Proof of Corollary 3.1. Note that $\beta(a)>-1$; hence, by Corollary 2.6.2 of [4] we have

$$
\frac{1}{m} \asymp \frac{a_{m}}{\sum_{n=1}^{m} a_{n}} \quad \text { as } m \rightarrow \infty .
$$

Since $\mu<\infty$, it is easy to see that

$$
\bar{F}(m)=o\left(\frac{1}{m}\right) \quad \text { as } m \rightarrow \infty .
$$

From this and (5.7), it follows that condition (3.1) is satisfied and, thus, by Theorem 3.1, the desired result is proved.

Proof of Corollary 3.2. Note that

$$
\beta\left(\left\{n^{-1-\beta} a_{n}\right\}\right)=-1-\beta+\beta(a)>-1 ;
$$

hence, by Theorem 2.6.1(b) of [4] we obtain

$$
\sum_{n=1}^{m} n^{-1-\beta} a_{n} \lesssim m^{-\beta} a_{m} \quad \text { as } m \rightarrow \infty
$$

So in view of $-1-\beta>0$ we have

$$
\frac{a_{m}}{\sum_{n=1}^{m} a_{n}} \geq \frac{a_{m}}{\sum_{n=1}^{m} n^{-1-\beta} a_{n}} \gtrsim \frac{a_{m}}{m^{-\beta} a_{m}}=m^{\beta} \quad \text { as } m \rightarrow \infty .
$$

From this and (3.3), we know that condition (3.1) is satisfied and, thus, by Theorem 3.1, the desired result is proved.

Proof of Corollary 3.3. We still let $\left\{b_{n}\right\}$ be as in Lemma 4.7. By Lemma 4.5, it is sufficient to prove (4.9). However, since $p_{m}=o\left(a_{m}\right)$, then by Lemma 4.8 and Lemma 4.9, we only need to prove that relation (4.9) holds with $b$ in place of $a$. Since $p_{m}=o\left(a_{m}\right)$, then by Lemma 4.9 we have, for every fixed $k \geq 1$,

$$
\sum_{n<k} b_{n} \mathrm{P}\left(S_{n}=m\right)=o\left(a_{m}\right)=o\left(b_{m}\right) \quad \text { as } m \rightarrow \infty .
$$


Therefore, it is easy to see that there exists a nonnegative integer-valued and nondecreasing function $k_{m}$ of $m$ with $k_{m} \rightarrow \infty$ as $m \rightarrow \infty$ such that

$$
\sum_{n<k_{m}} b_{n} \mathrm{P}\left(S_{n}=m\right)=o\left(b_{m}\right) \quad \text { as } m \rightarrow \infty .
$$

Hence, in order to prove that relation (4.9) holds with $b$ in place of $a$, we only need to prove that

$$
\sum_{k_{m} \leq n \leq \varepsilon m} b_{n} \mathrm{P}\left(S_{n}=m\right)=o\left(b_{m}\right), \quad m \rightarrow \infty, \varepsilon \rightarrow 0+.
$$

By the same reasoning as in the proof of Theorem 3.1 we know that relation (5.4) still holds with ' $\sum_{n \leq \varepsilon m}$ ' being replaced with ' $\sum_{k_{m} \leq n \leq \varepsilon m}$ ' and, simultaneously, we have (5.6). By (3.4) we have

$$
\bar{F}\left(\frac{m}{2 v_{0}}\right) \leq \bar{F}\left(\left[\frac{m}{2 v_{0}}\right]\right)=O\left(b_{\left[m / 2 v_{0}\right]}\right)=O\left(b_{m}\right) \quad \text { as } m \rightarrow \infty .
$$

From the condition $\sum_{n=1}^{\infty} a_{n}<\infty$ we have

$$
\sum_{n \geq k_{m}} b_{n} \rightarrow 0 \quad \text { as } m \rightarrow \infty
$$

Hence, by (5.9) we have

$$
I_{b}:=M \bar{F}\left(\frac{m}{2 v_{0}}\right) \sum_{k_{m} \leq n \leq \varepsilon m} b_{n} \leq M \bar{F}\left(\frac{m}{2 v_{0}}\right) \sum_{n \geq k_{m}} b_{n}=o\left(b_{m}\right) \quad \text { as } m \rightarrow \infty .
$$

From this and (5.6), it follows that

$$
\sum_{k_{m} \leq n \leq \varepsilon m}\left|b_{n}-b_{n+1}\right| \mathrm{P}\left(S_{n}>m\right)=o\left(b_{m}\right), \quad m \rightarrow \infty, \varepsilon \rightarrow 0+.
$$

Hence, by Lemma 4.6 we prove (5.8), as required.

Proof of Theorem 3.2. Here we use the same notation as in the proof of Lemma 4.5. We first assume that $\left\{a_{n}\right\}$ is nonincreasing eventually. Let $g(m):=G(m)-G(m-1)$. Then by Lemma 4.4 we have, for every fixed $\varepsilon>0$,

$$
\begin{aligned}
g(m) & \geq I I I_{a} \\
& \geq a\left(\frac{(1+\varepsilon) m}{\mu}\right) \sum_{m(1-\varepsilon) / \mu<n<m(1+\varepsilon) / \mu} \mathrm{P}\left(S_{n}=m\right) \\
& \sim \frac{a((1+\varepsilon) m / \mu)}{\mu} \quad \text { as } m \rightarrow \infty .
\end{aligned}
$$

Hence,

$$
\begin{aligned}
\limsup _{m \rightarrow \infty} \frac{a(m / \mu) / \mu}{g(m)} & \leq \limsup _{m \rightarrow \infty} \frac{a(m / \mu) / \mu}{g(m /(1+\varepsilon))} \limsup \frac{g(m /(1+\varepsilon))}{g(m)} \\
& \leq \limsup _{m \rightarrow \infty} \frac{g(m /(1+\varepsilon))}{g(m)} .
\end{aligned}
$$


Let $\varepsilon \downarrow 0$ in (5.10). Then by the regular oscillation of $g$ we prove that

$$
\limsup _{m \rightarrow \infty} \frac{a(m / \mu) / \mu}{g(m)} \leq 1 .
$$

Hence, in order to prove (3.2), we only need to prove that

$$
\liminf _{m \rightarrow \infty} \frac{a(m / \mu) / \mu}{g(m)} \geq 1 .
$$

It is easy to verify that relation (5.11) still holds if the integer variable $m$ is replaced by a real variable $x$. Moreover, without loss of generality, we assume that $g_{m}>0$ for all $m \geq 1$. Therefore, by (5.11), there exists a constant $\delta>0$ such that, for all $m \geq 1$,

$$
a_{m} \leq \delta g_{m}
$$

Then in view of

$$
I V_{a} \leq \delta \sum_{n \geq m(1+\varepsilon) / \mu} g_{n} \mathrm{P}\left(S_{n}=m\right),
$$

and by using the same reasoning as in the proof of Lemma 4.5 we prove that

$$
I V_{a}=o\left(g_{m}\right) \quad \text { as } m \rightarrow \infty .
$$

Similarly, we can prove that

$$
I_{a}=o\left(g_{m}\right) \quad \text { as } m \rightarrow \infty .
$$

By Lemma 4.4 we have

$$
\begin{aligned}
I I I_{a} & \leq a\left(\frac{(1-\varepsilon) m}{\mu}\right) \quad \sum_{m(1-\varepsilon) / \mu<n<m(1+\varepsilon) / \mu} \mathrm{P}\left(S_{n}=m\right) \\
& \sim \frac{a((1-\varepsilon) m / \mu)}{\mu} \quad \text { as } m \rightarrow \infty .
\end{aligned}
$$

Thus, if we can prove that

$$
I_{a}=o\left(g_{m}\right), \quad m \rightarrow \infty, \varepsilon \rightarrow 0+,
$$

then, similarly to the proof of (5.11), we can prove (5.12). By (5.13) we have

$$
I_{a} \leq \delta \sum_{n \leq \varepsilon m} g_{n} \mathrm{P}\left(S_{n}=m\right) .
$$

Note that condition (3.5) can be reformulated as

$$
\bar{F}(m)=o\left(\frac{G(m)-G(m-1)}{\sum_{n=1}^{m}(G(n)-G(n-1))}\right) \quad \text { as } m \rightarrow \infty .
$$

Hence, by the same reasoning as in the proof of Theorem 3.1 we prove that

$$
\sum_{n \leq \varepsilon m} g_{n} \mathrm{P}\left(S_{n}=m\right)=o\left(g_{m}\right), \quad m \rightarrow \infty, \varepsilon \rightarrow 0+,
$$

which together with (5.15), proves (5.14), as required. Since $g \in \mathfrak{C}$, then by (3.2) we prove that $a \in \mathfrak{C}$. If $\left\{a_{n}\right\}$ is nondecreasing eventually, the desired result follows similarly. 
Proof of Theorem 3.3. Since $\mu=\sum_{n=0}^{\infty} n p_{n}<\infty$, we have

$$
\sum_{n=0}^{\infty} n a_{n}<\infty
$$

Next we proceed as in the proof of Lemma 4.5. By condition (3.6) we know that, when $m$ is sufficiently large,

$$
I V_{a} \leq \sum_{n \geq m(1+\varepsilon) / \mu} p_{n} \mathrm{P}\left(S_{n}=m\right) \leq \sum_{n \geq m(1+\varepsilon) / \mu} p_{n} \mathrm{P}\left(S_{n} \leq \frac{n \mu}{1+\varepsilon}\right) .
$$

Then by the same reasoning to prove (4.12) we obtain, for every fixed $\varepsilon>0$,

$$
I V_{a}=o\left(p_{m}\right) \quad \text { as } m \rightarrow \infty .
$$

Moreover, by condition (3.6) and Theorem 2.0.8 of [4], we have, for every fixed $\varepsilon>0$,

$$
\begin{aligned}
I I_{a}+I I I_{a} & \leq \sup _{\varepsilon m<n<m(1+\varepsilon) / \mu} a_{n} \sum_{\varepsilon m<n \leq m(1+\varepsilon) / \mu} \mathrm{P}\left(S_{n}=m\right) \\
& \leq \frac{1}{1-p_{0}} \sup _{\varepsilon m<n<m(1+\varepsilon) / \mu} a_{n} \\
& =o\left(\sup _{\varepsilon m<n<m(1+\varepsilon) / \mu} p_{n}\right) \\
& =o\left(p_{m}\right) \quad \text { as } m \rightarrow \infty,
\end{aligned}
$$

where in the second step we have used Lemma 4.3. From Lemma 4.12 and (5.16), it follows that

$$
I_{a} \sim\left(\sum_{n=1}^{\infty} n a_{n}\right) p_{m}, \quad m \rightarrow \infty, \varepsilon \rightarrow 0+.
$$

Hence, from (4.10), the desired result follows.

Proof of Theorem 3.4. Following the same reasoning as in the proof of Theorem 3.3, we obtain (5.16). Then, from Lemma 4.12 and Lemma 4.5(ii), the desired result follows.

\section{Results for the nonarithmetic case}

In this section we always assume that $F$ is nonarithmetic. For any $h>0$, let

$$
p_{h}(x):=\mathrm{P}(X \in(x, x+h]) \text { for all } x>0 .
$$

Then results similar to that of the arithmetic case are presented as follows.

Theorem 6.1. Suppose that $a \in \mathfrak{C}$ and that

$$
\bar{F}(x)=o\left(\frac{a(x)}{\sum_{n=0}^{[x]} a_{n}}\right) \text { as } x \rightarrow \infty .
$$

Then, for every fixed $h>0$,

$$
G(x+h)-G(x) \sim \frac{a(x / \mu) h}{\mu} \text { as } x \rightarrow \infty .
$$


Corollary 6.1. Suppose that $a \in \mathfrak{C}$ and that $\beta(a)>-1$. Then relation (6.1) holds for every fixed $h>0$.

Corollary 6.2. Suppose that $a \in \mathfrak{C}, \beta(a) \leq-1$, and that there exists a constant $\beta<\beta(a)$ such that

$$
\bar{F}(x)=o\left(x^{\beta}\right) \text { as } x \rightarrow \infty .
$$

Then relation (6.1) holds.

Corollary 6.3. Let $h>0$. Suppose that $a \in \mathfrak{C}, \sum_{n=0}^{\infty} a_{n}<\infty, p_{h}(x)=o(a(x))$, and that

$$
\bar{F}(x)=O(a(x)) \text { as } x \rightarrow \infty .
$$

Then relation (6.1) holds.

Theorem 6.2. Let $h>0$. Suppose that $G(x+h)-G(x) \in \mathfrak{C},\left\{a_{n}\right\}$ is ultimately monotone, and that

$$
\bar{F}(x)=o\left(\frac{G(x+h)-G(x)}{G(x)}\right) \text { as } x \rightarrow \infty .
$$

Then $a \in \mathfrak{C}$ and relation (6.1) holds.

Theorem 6.3. Let $h>0$. Suppose that $p_{h} \in \mathfrak{D} \cap \mathfrak{L}, p_{h}$ is almost decreasing, $\rho_{F}>1$, and that

$$
a(x)=o\left(p_{h}(x)\right) \text { as } x \rightarrow \infty .
$$

Then $\sum_{n=1}^{\infty} n a_{n}<\infty$ and

$$
G(x+h)-G(x) \sim\left(\sum_{n=1}^{\infty} n a_{n}\right) p_{h}(x) \text { as } x \rightarrow \infty .
$$

Theorem 6.4. Let $h>0$. Suppose that $p_{h} \in \mathfrak{C}$, $p_{h}$ is almost decreasing, $\rho_{F}>1$, and that there exists a constant $K \geq 0$ such that

$$
a(x) \sim K p_{h}(x) \text { as } x \rightarrow \infty .
$$

Then $\sum_{n=1}^{\infty} n a_{n}<\infty$ and

$$
G(x+h)-G(x) \sim\left(\sum_{n=1}^{\infty} n a_{n}\right) p_{h}(x)+\frac{a(x / \mu) h}{\mu} \text { as } x \rightarrow \infty .
$$

All these results can be proved similarly to the corresponding results for the arithmetic case. However, with a little difference, we reformulate Lemma 4.3 as Lemma 6.1, below. Before stating it, we first note that, since $F(0)<1$, there must exist some $h_{0} \in(0, \infty)$ such that $F\left(h_{0}\right)<1$.

Lemma 6.1. For all $h \in(0, \infty)$ and $x>0$,

$$
\sum_{n=0}^{k} \mathrm{P}\left(S_{n} \in(x, x+h]\right) \leq \frac{h+h_{0}}{h_{0}\left(1-F\left(h_{0}\right)\right)} \mathrm{P}\left(S_{k} \geq x\right)
$$

and

$$
\sum_{n=k}^{\infty} \mathrm{P}\left(S_{n} \in(x, x+h]\right) \leq \frac{h+h_{0}}{h_{0}\left(1-F\left(h_{0}\right)\right)} \mathrm{P}\left(S_{k} \leq x+h\right) .
$$


Proof. In view of

$$
\begin{aligned}
& \left\{S_{n} \in\left(x, x+h_{0}\right]\right\} \\
& \quad=\left\{S_{n} \in\left(x, x+h_{0}\right], S_{n+1} \notin\left(x, x+h_{0}\right]\right\} \cup\left\{S_{n} \in\left(x, x+h_{0}\right], S_{n+1} \in\left(x, x+h_{0}\right]\right\},
\end{aligned}
$$

we have

$$
\begin{aligned}
\sum_{n=0}^{k} \mathrm{P}\left(S_{n} \in\left(x, x+h_{0}\right]\right)= & \sum_{n=0}^{k} \mathrm{P}\left(S_{n} \in\left(x, x+h_{0}\right], S_{n+1} \notin\left(x, x+h_{0}\right]\right) \\
& +\sum_{n=0}^{k} \mathrm{P}\left(S_{n} \in\left(x, x+h_{0}\right], S_{n+1} \in\left(x, x+h_{0}\right]\right) \\
:= & I_{h}+I I_{h} .
\end{aligned}
$$

Since $\left\{S_{n} \in\left(x, x+h_{0}\right], S_{n+1} \notin\left(x, x+h_{0}\right]\right\}$ for $n=0,1, \ldots, k$ is a collection of disjoint events, we have

$$
\begin{aligned}
I_{h} & =\mathrm{P}\left(\bigcup_{n=0}^{k}\left\{S_{n} \in\left(x, x+h_{0}\right], S_{n+1} \notin\left(x, x+h_{0}\right]\right\}\right) \\
& \leq \mathrm{P}\left(\bigcup_{n=0}^{k}\left\{S_{n} \in\left(x, x+h_{0}\right]\right\}\right) \\
& \leq \mathrm{P}\left(S_{k} \geq x\right)
\end{aligned}
$$

where the last step holds since $X$ is nonnegative. Note that

$$
\left\{S_{n} \in\left(x, x+h_{0}\right], S_{n+1} \in\left(x, x+h_{0}\right]\right\} \subset\left\{S_{n} \in\left(x, x+h_{0}\right], X_{n+1} \leq h_{0}\right\},
$$

and that $\left\{S_{n} \in\left(x, x+h_{0}\right]\right\}$ and $\left\{X_{n+1} \leq h_{0}\right\}$ are independent. Then we obtain

$$
\begin{aligned}
I I_{h} & \leq \sum_{n=0}^{k} \mathrm{P}\left(S_{n} \in\left(x, x+h_{0}\right], X_{n+1} \leq h_{0}\right) \\
& =\sum_{n=0}^{k} \mathrm{P}\left(S_{n} \in\left(x, x+h_{0}\right]\right) \mathrm{P}\left(X_{n+1} \leq h_{0}\right) \\
& =F\left(h_{0}\right) \sum_{n=0}^{k} \mathrm{P}\left(S_{n} \in\left(x, x+h_{0}\right]\right) .
\end{aligned}
$$

Combining (6.4), (6.5), and (6.6) gives

$$
\sum_{n=0}^{k} \mathrm{P}\left(S_{n} \in\left(x, x+h_{0}\right]\right) \leq \mathrm{P}\left(S_{k} \geq x\right)+F\left(h_{0}\right) \sum_{n=0}^{k} \mathrm{P}\left(S_{n} \in\left(x, x+h_{0}\right]\right),
$$

which implies that

$$
\sum_{n=0}^{k} \mathrm{P}\left(S_{n} \in\left(x, x+h_{0}\right]\right) \leq \frac{1}{1-F\left(h_{0}\right)} \mathrm{P}\left(S_{k} \geq x\right)
$$


For any $h \in(0, \infty)$, let $l:=\left[h / h_{0}\right]$. Then by (6.7) we obtain

$$
\begin{aligned}
\sum_{n=0}^{k} \mathrm{P}\left(S_{n} \in(x, x+h]\right) & \leq \sum_{n=0}^{k} \sum_{j=0}^{l} \mathrm{P}\left(S_{n} \in\left(x+j h_{0}, x+(j+1) h_{0}\right]\right) \\
& =\sum_{j=0}^{l} \sum_{n=0}^{k} \mathrm{P}\left(S_{n} \in\left(x+j h_{0}, x+(j+1) h_{0}\right]\right) \\
& \leq \frac{1}{1-F\left(h_{0}\right)} \sum_{j=0}^{l} \mathrm{P}\left(S_{k} \geq x+j h_{0}\right) \\
& \leq \frac{1}{1-F\left(h_{0}\right)} \sum_{j=0}^{l} \mathrm{P}\left(S_{k} \geq x\right) \\
& =\frac{l+1}{1-F\left(h_{0}\right)} \mathrm{P}\left(S_{k} \geq x\right) \\
& \leq \frac{h+h_{0}}{h_{0}\left(1-F\left(h_{0}\right)\right)} \mathrm{P}\left(S_{k} \geq x\right),
\end{aligned}
$$

completing the proof of (6.2). The proof of (6.3) is similar.

\section{Concluding remarks}

(a) Obviously, Corollary 3.1 generalizes both Theorem 1(a) of [6] and Corollary 2 of [17]. Moreover, if $\left\{a_{n}\right\}$ is nonincreasing then we have

$$
\sum_{n=1}^{m} a_{n} \leq m a_{1}
$$

and, thus, the relation

$$
\bar{F}(m)=o\left(\frac{a_{m}}{m}\right) \quad \text { as } m \rightarrow \infty,
$$

implies (3.1). From this we know that Theorem 7 of [17] is a special case of Theorem 3.1.

(b) Theorem 2(c) of [6] states that if $a \in R V(\alpha)$ with $\alpha<-1$ and

$$
\bar{F}(m) \sim K a_{m} \text { for some } K \geq 0,
$$

then relation (3.2) holds. We assert that the result above is generalized by Corollary 3.3. To see this, we only need to note that (7.1) and $a \in \mathfrak{L}$ imply that

$$
\frac{p_{m}}{a_{m}}=\frac{\bar{F}(m)-\bar{F}(m+1)}{a_{m}}=\frac{\bar{F}(m)}{a_{m}}-\frac{\bar{F}(m+1)}{a_{m+1}} \frac{a_{m+1}}{a_{m}} \rightarrow K-K=0 \quad \text { as } m \rightarrow \infty .
$$

(c) Obviously, the second part of Theorem 2(b) of [6] is implied by Corollary 3.2. However, for harmonic measures, condition (3.1) reduces to

$$
\bar{F}(m)=o\left(\frac{1}{m \log m}\right) .
$$


However, the first part of Theorem 2(b) of [6] only requires that

$$
\bar{F}(m)=o\left(\frac{1}{m}\right),
$$

which is weaker than (7.2) and is satisfied when $\mu<\infty$.

(d) Theorem 6.2.2 of [18], together with their Theorem 6.2.1, implies that if $F$ is nonarithmetic, $\mu<\infty, a \in \mathfrak{C}$, and that, for all $n \geq 1$,

$$
\frac{a_{n+1}}{a_{n}} \geq \frac{n}{n+1}
$$

then relation (6.1) holds. Note that condition (7.3) implies that, for every fixed $\lambda>1$ and all $n \geq 1$,

$$
\frac{a(\lambda n)}{a_{n}} \geq \frac{1}{\lambda}
$$

and, thus, $\beta(a) \geq-1$. Thus, we cannot conclude that the result mentioned above is implied by Corollary 6.1 since it includes the case in which $\beta(a)=-1$.

(e) Consider the case of subordination, i.e. $\sum_{n=0}^{\infty} a_{n}=1$. Then, by taking sums, any result in this paper implies a result for $\bar{G}$. However, we think it is more suitable to consider the behavior of $\bar{G}$ directly. In fact, with some modifications to the proofs (for example, using the large deviation inequality for $\mathfrak{D} \cap \mathfrak{L}$ in [20] or the precise large deviation relation for $\mathfrak{C}$ in [16] rather than those for $R V$, etc.), Proposition 4.1, Proposition 4.3, and Lemma 4.7 of [7] can be extended from the setting of regular variation to more general cases.

\section{Acknowledgement}

The author would like to thank the anonymous referee for his/her helpful comments and suggestions.

\section{References}

[1] Alsmeyer, G. (1992). On generalized renewal measures and certain first passage times. Ann. Prob. 20, 1229-1247.

[2] Asmussen, S., Foss, S. And Korshunov, D. (2003). Asymptotics for sums of random variables with local subexponential behaviour. J. Theoret. Prob. 16, 489-518.

[3] Berman, S. M. (1992). The tail of the convolution of densities and its application to a model of HIV-latency time. Ann. Appl. Prob. 2, 481-502.

[4] Bingham, N. B., Goldie, C. M. And Teugels, J. L. (1987). Regular Variation (Encyclopedia Math. Appl. 27). Cambridge University Press.

[5] Chover, J., Ney, P. And Wainger, S. (1973). Functions of probability measures. J. Analyse Math. 26, $255-302$.

[6] Embrechts, P., Maejima, M. And Omey, E. (1984). A renewal theorem of Blackwell type. Ann. Prob. 12, 561-570.

[7] Fä̈, G., González-Arévalo, G., Mikosch, T. and Samorodnitsky, G. (2006). Modeling teletraffic arrivals by a Poisson cluster process. Queueing Systems 54, 121-140.

[8] Feller, W. (1971). An Introduction to Probability Theory and Its Applications, Vol. II, 2nd edn. John Wiley, New York.

[9] FuK, D. H. And Nagaev, S. V. (1971). Probabilistic inequalities for sums of independent random variables. Theory Prob. Appl. 16, 660-675.

[10] Hilberdink, T. (1996). On the Taylor coefficients of the composition of two analytic functions. Ann. Acad. Scientiarum Fenn. Math. 21, 189-204.

[11] Kalma, J. M. (1972). Generalized renewal measures. Doctoral Thesis, Groningen University. 
[12] Karlin, S. and Taylor, H. M. (1975). A First Course in Stochastic Processes, 2nd edn. Academic Press, New York.

[13] Kawata, T. (1961). A theorem of renewal type. Kōdai Math. Sem. Rep. 13, 185-194.

[14] Lin, J. (2008). A one-sided large deviation local limit theorem. Statist. Prob. Lett. 78, 2679-2684.

[15] Mallor, F. AND OMey, E. (2006). Univariate and multivariate weighted renewal theory. In Collection of Monographies of the Department of Statistics and Operations Research, Vol. 2. Public University of Navarra, Pamplona, Spain.

[16] NG, K. W., TANG, Q., YAn, J. And YAng, H. (2004). Precise large deviations for sums of random variables with consistently varying tails. J. Appl. Prob. 41, 93-107.

[17] Omey, E. And Teugels, J. (2002). Weighted renewal functions: a hierarchical approach. Adv. Appl. Prob. 34, 394-415.

[18] Rolski, T., Schmidli, H., Schmidt, V. And Teugels, J. (1999). Stochastic Processes for Insurance and Finance. John Wiley, Chichester.

[19] Stam, A. J. (1973). Regular variation of the tail of a subordinated probability distribution. Adv. Appl. Prob. 5, 308-327.

[20] TANG, Q. AND YAN, J. (2002). A sharp inequality for the tail probabilities of sums of i.i.d. r.v.'s with dominatedly varying tails. Sci. China Ser. A 45, 1006-1011. 